\title{
Synchronization Transition and Traffic Congestion in One-Dimensional Traffic Model
}

\author{
Zhi Xin ${ }^{1,2}$ and Jian $X u^{1}$ \\ ${ }^{1}$ School of Aerospace Engineering and Applied Mechanics, Tongji University, Shanghai 200092, China \\ ${ }^{2}$ Mathematics Science College, Inner Mongolia Normal University, Hohhot 010022, China \\ Correspondence should be addressed to Jian Xu; xujian@tongji.edu.cn
}

Received 23 June 2014; Accepted 28 August 2014

Academic Editor: Yongli Song

Copyright (C) 2015 Z. Xin and J. Xu. This is an open access article distributed under the Creative Commons Attribution License, which permits unrestricted use, distribution, and reproduction in any medium, provided the original work is properly cited.

A nonlinear car-following model with driver's reaction time is studied from the synchronization transition viewpoint. We investigate the traffic congestion from the view of chaos system synchronization transition. Our result shows that the uniform flow corresponds to the complete synchronization and the stop-and-go congested state corresponds to the lag synchronization of the vehicles. An analytical criterion for synchronization manifolds stability is obtained; the analytical result and the numerical result are consistent. The synchronization transition is also trigged by the driver's reaction time. We analyze the car-following model by the use of the nonlinear analysis method and derive the modified $\mathrm{KdV}$ equation describing the kink density wave.

\section{Introduction}

Traffic jams are very annoying in our life and have been studied by many physicists [1]; however, the precise mechanism for generation and propagation of traffic jams is still not clearly understood. Some traffic models have been established to study the dynamics of traffic flow, such as car-following models, cellular automaton models, gas kinetic models, and hydrodynamic models [2].

Car-following models or microscopic models describe the behaviors of individual vehicles, which are described by ordinary differential equation or delay differential equation. Recently, one more widely used car-following model is the optimal velocity model $[3,4]$; by this kind of model, the effects of fluctuations of traffic jams are analyzed, the jamming transitions and density waves have been invested [5-9], and the bifurcation phenomena of the oscillating solution are also explored by using numerical continuations techniques [1012]. Another popular car-following model is the intelligent driver model that was introduced by Treiber et al. $[13,14]$; in this model, all parameters have a clear physical meaning; congested traffic states have been empirically observed and microscopically simulated. Many models are able to explain uniform flow as well as stop-and-go waves; however, the transition [15-28] between the two qualitatively different solutions is still not clarified.

According to the car-following model theory, for each individual vehicle, an equation of motion is the analogue of Newton's equation for each individual particle in a system of interacting classical particles. In Newtonian mechanics, the acceleration may be regarded as the response of the particle to the stimulus, and it receives in the form of force which includes both the external force and those arising from its interaction with the other particles in the system. In traffic system, each driver can respond to the surrounding traffic conditions only by accelerating or decelerating the vehicle; so, the basic philosophy of the car-following theories can be summarized by the equation [Response $]_{k}-[\text { Stimulus }]_{k}$ for the $k$ th vehicle $(k=1,2, \ldots)$.

We know, for a chaotic system, the appearances and robustness of chaotic synchronization states have been established by means of different coupling schemes [29], one of which is Pecora and Carroll method (unidirectional coupling or drive-response coupling).

In this paper, we regard the car-following system as a drive-response coupling chaotic system; we make use of 
chaotic systems synchronization transition [30-34] method to study the synchronization transition of microscopic movement of the vehicles and further reveal the relationship between synchronization transition and traffic congestion. First, we use the long wave expansion method to give an analytical criterion for synchronization manifolds stability. In order to verify the analytical result, we use DDE-BIFTOOL to perform a two-parameter bifurcation analysis of the model; due to the demands on the CPU time and the memory, the investigation was restricted to the setting of $N=15$ vehicles, and the analytical result is consistent with the numerical result. Second, we find different transition region in twoparameter plane and the vehicles display different motion. Third, we consider how the driver's reaction time impacts on the synchronization transition. Finally, we analyze the carfollowing model by the use of the nonlinear analysis method and we derive the modified $\mathrm{KdV}$ equation describing the kink density wave.

The layout of this paper is organized as follows. We introduce the car-following model in Section 2 and derive analytical criteria for synchronization manifolds stability in Section 3. Numerical simulation is in Section 4. Nonlinear analysis is in Section 5. In Section 6, we present the main results.

\section{Car-Following Model}

Here, we consider a single lane of traffic with identical vehicles; displacements and velocities are denoted as $x_{k}(t)$ and $v_{k}(t)$, respectively; the spacing of adjacent vehicle is called headway $\left(h_{k}(t)\right)$. For the sake of simplicity, we suppose that $N$ vehicles are placed on a circular road of length $\left(\sum_{k=1}^{N} h_{k}=L\right)$. The headway consists of the condition

$$
\dot{h}_{k}(t)=v_{k+1}(t)-v_{k}(t)
$$

The acceleration of the $k$ th vehicle is given by

$$
\dot{v}_{k}(t)=\alpha\left[V\left(h_{k}(t-\tau)\right)-v_{k}(t)\right]
$$

The $\alpha$ is called the sensitivity of the vehicles and the $\tau$ is driver's reaction time. The function $V(h)$ is optimal velocity function; it has the following properties.

(a) $V(h)$ is a nonnegative, continuous, and monotone increasing function.

(b) $V(h) \rightarrow v_{0}$ as $h \rightarrow \infty$.

(c) There exists a jam headway $h_{\text {stop }}$ such that $V(h)=0$ for $h \in\left[0, h_{\text {stop }}\right]$.

The dimensional parameter OV function [12] is

$$
V(h)= \begin{cases}0, & h \leq h_{\text {stop }}, \\ v_{0} \frac{\left(h-h_{\text {stop }}\right)^{3}}{h_{\text {stop }}^{3}+\left(h-h_{\text {stop }}\right)^{3}}, & h>h_{\text {stop }} .\end{cases}
$$

We introduce the rescaled variables $\widehat{h}=h / h_{\text {stop }}, \widehat{t}=$ $v_{0}\left(t / h_{\text {stop }}\right)$; the OV function becomes

$$
V(\widehat{h})= \begin{cases}0, & \widehat{h} \leq 1, \\ \frac{(\widehat{h}-1)^{3}}{1+(\widehat{h}-1)^{3}}, & \widehat{h}>1 .\end{cases}
$$

Model (1) and model (2) are transformed into

$$
\begin{gathered}
\dot{\hat{h}}_{k}(\widehat{t})=\widehat{v}_{k+1}(\widehat{t})-\widehat{v}_{k}(\widehat{t}), \\
\dot{\widehat{v}}_{k}(\widehat{t})=\widehat{\alpha}\left[\widehat{V}\left(\widehat{h}_{k}(\widehat{t}-\widehat{\tau})\right)-\widehat{v}_{k}(\widehat{t})\right] .
\end{gathered}
$$

Here $\widehat{\alpha}=\alpha h_{\text {stop }} / v_{0}, \widehat{\tau}=\tau v_{0} / h_{\text {stop }}$; in the remainder of this paper we take the rescaled OV function and model. In order to express simplicity, the OV function and model are expressed:

$$
\begin{gathered}
V(h)= \begin{cases}0, & h \leq 1, \\
\frac{(h-1)^{3}}{1+(h-1)^{3}}, & h>1,\end{cases} \\
\dot{h}_{k}(t)=v_{k+1}(t)-v_{k}(t), \\
\dot{v}_{k}(t)=\alpha\left[V\left(h_{k}(t-\tau)\right)-v_{k}(t)\right] .
\end{gathered}
$$

\section{Synchronization Transition}

The synchronization manifold of the system (7) and (8) is

$$
X^{1}\left(h_{1}, v_{1}\right)=X^{2}\left(h_{2}, v_{2}\right)=\cdots=X^{N}\left(h_{N}, v_{N}\right) .
$$

The system $((7),(8))$ possesses uniform flow equilibrium

$$
\begin{gathered}
h_{1}=h_{2}=\cdots=h_{N}=h^{*}=\frac{L}{N}, \\
v_{1}=v_{2}=\cdots=v_{N}=v^{*}=V\left(h^{*}\right) .
\end{gathered}
$$

All vehicles reach complete synchronization. The stability of the synchronization manifolds will change when the parameter $h^{*}$ is varied. To see whether the synchronization manifold of the system $((7),(8))$ is stable or not, we add a small perturbation

$$
h_{k}(t)=h^{*}+\widetilde{h}_{k}(t), \quad v_{k}(t)=v^{*}+\widetilde{v}_{k}(t) .
$$

According to (7) and (8), we can calculate a linearized equation with respect to the uniform flow (10). The linearized equation is

$$
\begin{gathered}
\dot{\widetilde{h}}_{k}(t)=\widetilde{v}_{k+1}(t)-\widetilde{v}_{k}(t), \\
\dot{\widetilde{v}}_{k}(t)=\alpha V^{\prime}\left(h^{*}\right) \widetilde{h}_{k}(t-\tau)-\alpha \widetilde{v}_{k}(t) .
\end{gathered}
$$

Indeed, we have $\tilde{h}_{k}(t)=\tilde{x}_{k+1}(t)-\tilde{x}_{k}(t), \dot{\tilde{x}}_{k+1}(t)=\widetilde{v}_{k}(t)$, and $\dot{\widetilde{h}}_{k+1}(t)=\widetilde{v}_{k+1}(t)-\widetilde{v}_{k}(t)$. 


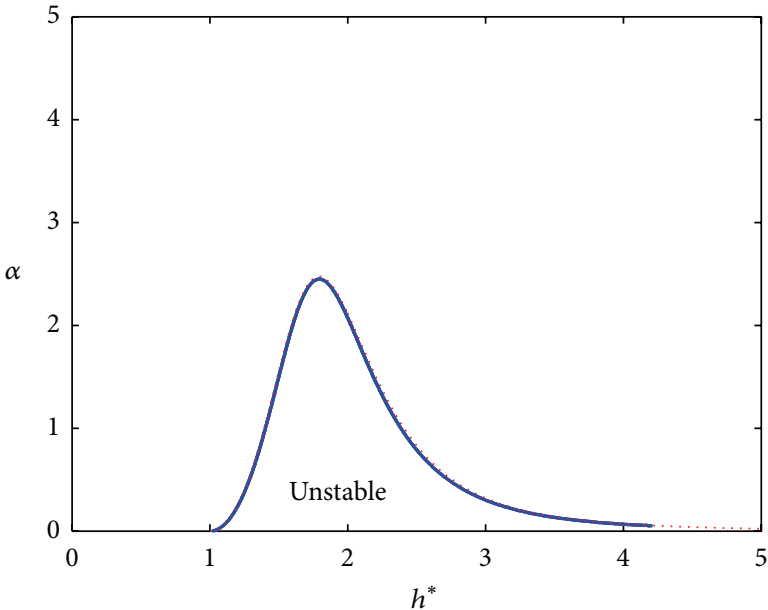

Analytical stability curve

Numerical continuation Hopf $(j=1)$ curve

(a)

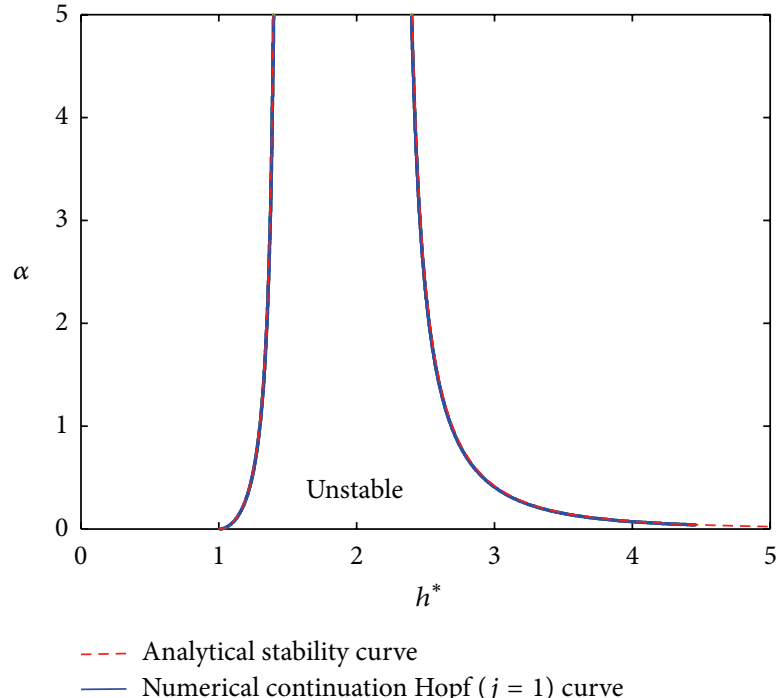

(b)

FIGURE 1: Stability diagram in the $\left(h^{*}, \alpha\right)$ plane for (a) $\tau=0.2$ and for (b) $\tau=1$.

Orosz et al. have used the dynamical system approach and numerical continuation technique to give out the stable curves, but the numerical continuation method (DDEBIFTOOL) needs a lot of CPU time and the memory; below, we can find a simple method to analyze the linear stability of the synchronization manifold [10].

Physical Approach. Let $\tilde{x}_{k}(t)=A e^{i k \theta+\lambda t}, \lambda \in C, \theta \in(0,2 \pi)$; the characteristic equation is given by

$$
\left[\lambda^{2}+\alpha \lambda+\alpha V^{\prime}\left(h^{*}\right) e^{-\lambda \tau}\right]^{N}-\left[\alpha V^{\prime}\left(h^{*}\right) e^{i \theta k-\lambda \tau}\right]^{N}=0
$$

We know that the leading term of $\lambda$ is of order $i \theta$. When $i \theta \rightarrow$ $0, \lambda \rightarrow 0$. We can derive the long wave expansion of $\lambda$, which is determined order by order around $i \theta \approx 0$. By expanding

$$
\lambda=\lambda_{1}(i \theta)+\lambda_{2}(i \theta)^{2}+\lambda_{3}(i \theta)^{3}+\cdots,
$$

the first- and second-order terms of $\lambda$ are obtained:

$$
\begin{gathered}
\lambda_{1}=V^{\prime}\left(h^{*}\right), \\
\lambda_{2}=\frac{V^{\prime}\left(h^{*}\right)}{2}\left(1-2 \tau V^{\prime}\left(h^{*}\right)-2 \frac{V^{\prime}\left(h^{*}\right)}{\alpha}\right) .
\end{gathered}
$$

If $\lambda_{2}$ is a positive value, the synchronization manifold is stable; if $\lambda_{2}$ is a negative value, the synchronization manifold is unstable.

By $\lambda_{2}>0$, we get

$$
1-2 \tau V^{\prime}\left(h^{*}\right)-2 \frac{V^{\prime}\left(h^{*}\right)}{\alpha}>0 .
$$

Equation (16) is the analytical criterion for synchronization manifold stability.
If the number of the vehicles is large, to obtain the stable boundary curve by the numerical continuations method requires a lot of time. In order to compare with numerical method, we now focus on the case of $N=15$ vehicles. For $\tau>0$, the stable boundary curve is shown in Figure 1. We use the numerical continuation techniques (DDEBIFTOOL) to get the blue curve and we get the analytical curve (red curve) according to (16). We can find that the blue curve and the red curve completely overlap, and this shows that our analytical approach is correct. In Section 4 numerical simulations also verify our conclusion.

When the stability of the synchronization manifold is lost, we can get $\widetilde{x}_{k}(t)=A \sin (\omega t+(2 j \pi / N) k), \widetilde{v}_{k}(t)=A \omega \cos (\omega t+$ $(2 j \pi / N) k), 2 j \pi / N=\theta$, and $\widetilde{v}_{k}(t)=\widetilde{v}_{k+1}\left(t-\left(T_{\text {per }} / N\right)\right)$, and $T_{\text {per }}=2 j \pi / \omega$ is the period. For two adjacent vehicles, the speed of the two vehicles has a certain time delay; the vehicles will get lag synchronization.

Figure 1(a) shows that the synchronization manifold is stable above the sable boundary curve and the vehicles can achieve complete synchronization; the synchronization manifold is unstable below the stable boundary curve and the vehicles can achieve lag synchronization. The synchronization transition of the vehicles arises when the sensitivity $(\alpha)$ is fixed and the headway $\left(h^{*}\right)$ varies from the right to the left through the critical curve. Figure 1(b) shows that the unstable region is unbounded for $\tau>0.595$.

Below we focus on the synchronization transition of the vehicles. If $0 \leq \tau \leq 0.595$, according to Figure 2 , by adding a vertical line across the top point $((1.79,2.53))$ of the critical curve, we can divide the whole region into three regions, region I, region II, and region III. Region I and region II are stable; region III is unstable. If $\tau>0.595$, the region is divided analogous to the situation when $0 \leq \tau \leq 0.595$, as is shown in Figure 1(b). 


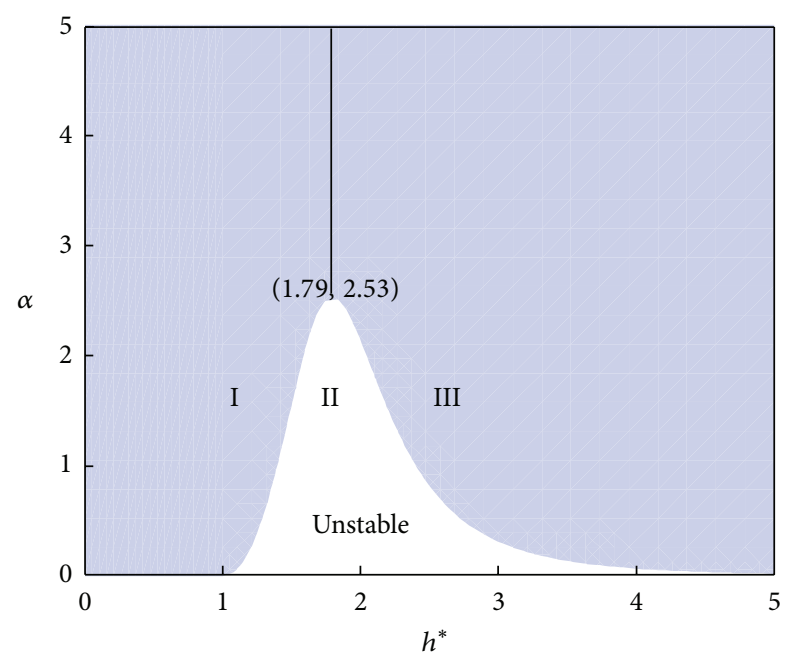

FIGURE 2: The synchronization transition area.

\section{Numerical Simulation}

In Figure 3, we show the space-time evolutions of the vehicles' velocity $\left(v_{i}\right)$ and location $\left(x_{i}\right)$ plotted against time $(t)$. We consider the velocity of the first and the second vehicles, the location of fifteen vehicles. First, we take a point in region III where $\tau=0.2, \alpha=0.5, h^{*}=4, v=0.97$, and $N=15$. The corresponding velocity-time plot and the space-time plot are shown in Figures 3(a) and 3(b). In region III, the headway is larger and the length of the road is longer; all vehicles travel with the same high speed. This is a freely moving phase. Second, we take a point in region II where $\tau=0.2, \alpha=0.5$, $h^{*}=2, v=0.5$, and $N=15$. The corresponding velocitytime history plot and the space-time plot are shown in Figures 3(c) and 3(d). The vehicles travel with stop-and-go state and get lag synchronization; the velocity of the adjacent vehicles has a certain time delay. Last, we take a point in region I where $\tau=0.2, \alpha=0.5, h^{*}=1.2, v=0.008$, and $N=15$. Figures $3(\mathrm{e})$ and $3(\mathrm{f})$ show the velocity-time history plot and the spacetime relation; the vehicles travel with a low speed close to zero and get complete synchronization, that is, a uniformly congested phase.

In Figure 4 the stability under different time delay influence is compared. We know that the unstable region is bounded for $0 \leq \tau<3 / 4 \sqrt[3]{2} \approx 0.595$; when $\tau>0.595$, the unstable region is unbounded. We find that the unstable region becomes larger as the time delay increases.

In Figure 5 we consider the time delay effect on the synchronization transition. For $\tau=0$, the vehicles travel with the same speed and they get complete synchronization. For $\tau=0.4$, the vehicles get lag synchronization and travel with moderate amplitude. If the time delay is increased to 1 , the vehicles travel with large amplitude. If the time delay continues to increase, the traffic is completely congested.

According to the analytical stability condition (23), the stability of the synchronization manifold is strictly dependent on the three parameters $\left(\alpha, \tau, h^{*}\right)$.

\section{Nonlinear Analysis}

We analyze the car-following model by the use of the nonlinear analysis method. We derive the modified $\mathrm{KdV}$ equation describing the kink density wave. We now consider long-wavelength models in the traffic flow on coarse-grained scales. The simplest way to describe the long-wavelength models is the long wave expansion. We consider the slowly varying behavior at long wavelengths near the critical point $\left(h_{c}^{*}, \alpha_{c}\right)$. We assume that the value of $v_{k}(t)$ is determined adiabatically by $h_{k}$. This statement is expressed by the relation

$$
v_{k}(t)=v\left(h_{k}, h_{k+1}, h_{k-1}, \ldots\right) .
$$

We define the headway as

$$
h_{k}(t)=h_{c}^{*}+\varepsilon \tilde{h}(Z, T),
$$

and $Z$ and $T$ are a scaled position and time defined by

$$
Z=\varepsilon(k+b t), \quad T=\varepsilon^{3} p t .
$$

Then, from (18), $v_{k}(t)$ is expressed as

$$
v_{k}(t)=v(\varepsilon \tilde{h}(Z, T), \varepsilon \widetilde{h}(Z+2 \varepsilon, T), \varepsilon \tilde{h}(Z-2 \varepsilon, T), \ldots) .
$$

Since $\varepsilon$ is a small parameter, a Taylor expansion can be applied to terms like $\widetilde{h}(Z+2 \varepsilon, T)$ in (20). This leads to the expression

$$
v_{k}(t)=\widetilde{v}\left(\varepsilon \widetilde{h}(Z, T), \varepsilon^{2} \partial_{Z} \widetilde{h}(Z, T), \varepsilon^{3} \partial_{Z}^{2} \widetilde{h}(Z, T), \ldots\right) .
$$

Here, $\widetilde{v}$ remains unknown; we determine it by assuming the form

$$
\widetilde{v}=\sum_{n=0}^{\infty} \varepsilon^{n} \widetilde{v}^{(n)}\left(\widetilde{h}(Z, T), \partial_{Z} \widetilde{h}(Z, T), \partial_{Z}^{2} \widetilde{h}(Z, T), \ldots\right) .
$$

From the form of (22), we find the terms $\widetilde{v}^{(n)}$ are expressed as

$$
\begin{aligned}
& {\widetilde{v}^{(0)}}^{=} \widetilde{v}_{0} \\
& \widetilde{v}^{(1)}=\widetilde{v}_{1} \widetilde{h} \\
& \widetilde{v}^{(2)}=\widetilde{v}_{20} \widetilde{h}^{2}+\widetilde{v}_{21} \partial_{Z} \tilde{h} \\
& \widetilde{v}^{(3)}=\widetilde{v}_{30} \widetilde{h}^{3}+\widetilde{v}_{31} \partial_{Z} \widetilde{h}^{2}+\widetilde{v}_{32} \partial_{Z}^{2} \widetilde{h} \\
& \widetilde{v}^{(4)}=\widetilde{v}_{40} \widetilde{h}^{4}+\widetilde{v}_{41} \partial_{Z} \widetilde{h}^{3}+\widetilde{v}_{42} \partial_{Z}^{2} \widetilde{h}^{2}+\widetilde{v}_{43} \partial_{Z}^{3} \widetilde{h}
\end{aligned}
$$

where $\widetilde{v}_{0}, \widetilde{v}_{1}, \widetilde{v}_{20}, \widetilde{v}_{21}, \widetilde{v}_{30}, \widetilde{v}_{31}, \widetilde{v}_{32}, \widetilde{v}_{40}, \widetilde{v}_{41}, \widetilde{v}_{42}, \widetilde{v}_{43}$, and so forth are constants, which are calculated in the following way. First, we substitute (22) (using (23)) into (8), where $\dot{v}_{k}$ is expressed as a function of $\widetilde{h}$,

$$
\begin{aligned}
\dot{v}_{k}= & \varepsilon^{2} \widetilde{v}_{1} b \partial_{Z} \widetilde{h}+\varepsilon^{3}\left(b \widetilde{v}_{20} \widetilde{h}^{2}+b \widetilde{v}_{21} \partial_{Z}^{2} \widetilde{h}\right) \\
& +\varepsilon^{4}\left(p \widetilde{v}_{1} \partial_{T} \widetilde{h}+b \widetilde{v}_{30} \widetilde{h}^{3}+b \widetilde{v}_{31} \partial_{Z}^{2} \widetilde{h}^{2}+b \widetilde{v}_{32} \partial_{Z}^{3} \widetilde{h}\right) \\
& +\varepsilon^{5}\left(p \widetilde{v}_{20} \partial_{T} \widetilde{h}^{2}+p \widetilde{v}_{21} \partial_{Z, T} \widetilde{h}+b \widetilde{v}_{40} \widetilde{h}^{4}\right. \\
& \left.+b \widetilde{v}_{41} \partial_{Z}^{2} \widetilde{h}^{3}+b \widetilde{v}_{42} \partial_{Z}^{3} \widetilde{h}^{2}+b \widetilde{v}_{43} \partial_{Z}^{4} \widetilde{h}\right)+\cdots
\end{aligned}
$$




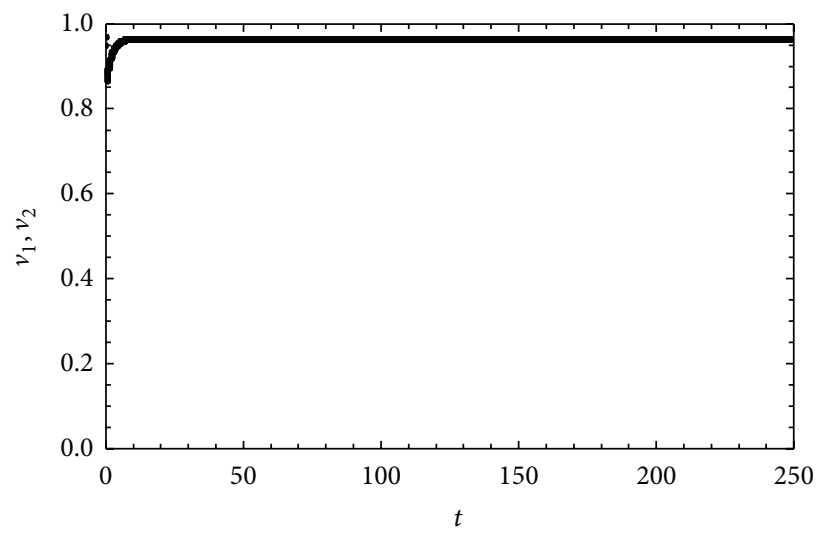

(a)

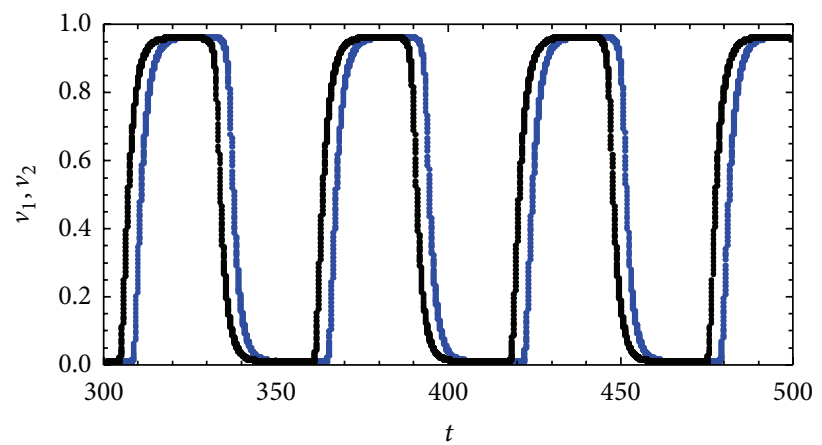

(c)

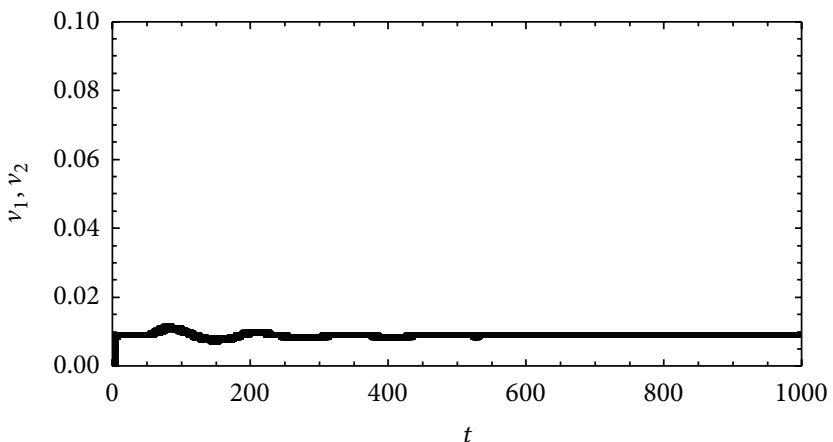

(e)

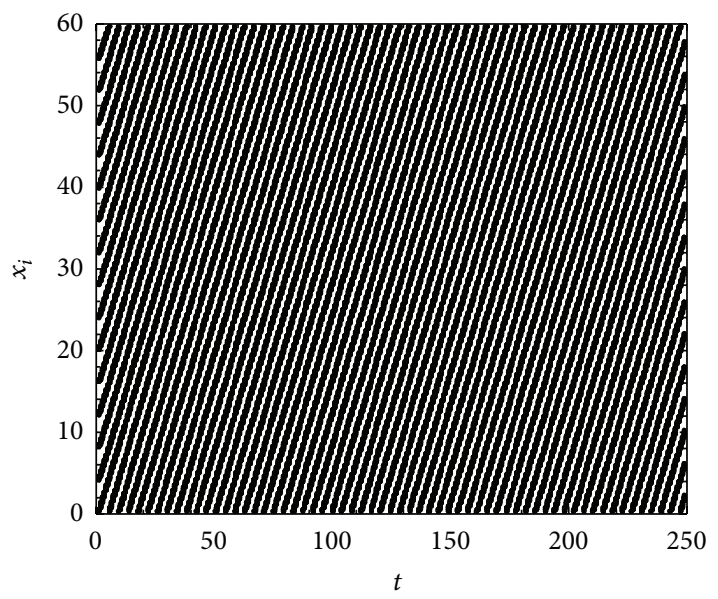

(b)

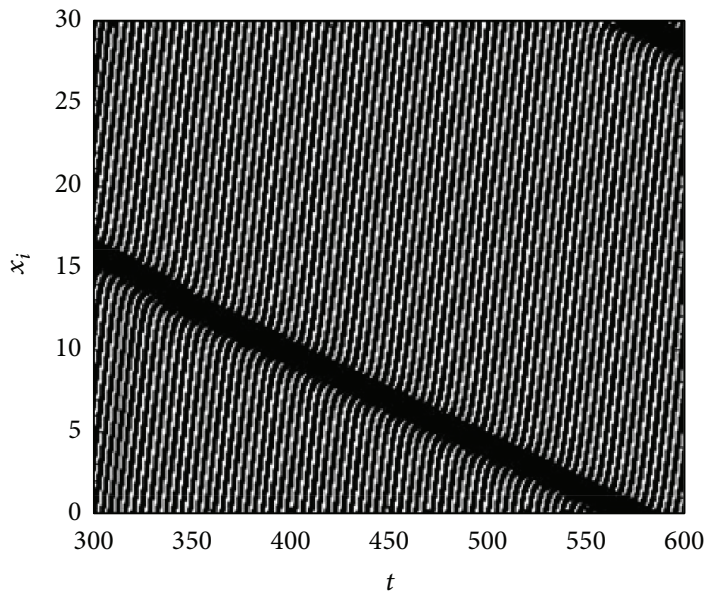

(d)

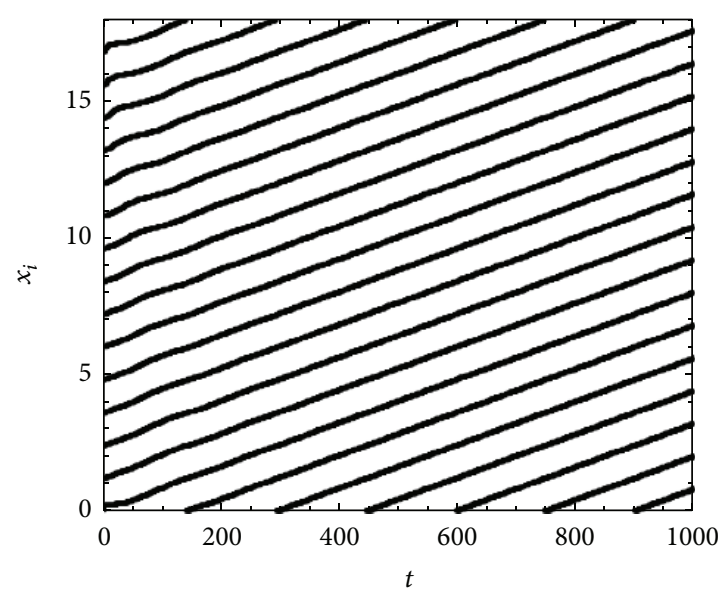

(f)

FIgURE 3: Plot of the velocity and the location of vehicles against time in different regions. We consider the speeds of the first two cars and the location of fifteen cars. The blue curve represents the speed of the first car and the black curve represents the speed of the second car. (a) and (b): region III, $\tau=0.2, \alpha=0.5, h^{*}=4, v=0.97$. (c) and (d): region II, $\tau=0.2, \alpha=0.5, h^{*}=2, v=0.5$. (e) and (f): region I, $\tau=0.2$, $\alpha=0.5, h^{*}=1.2, v=0.008$. 


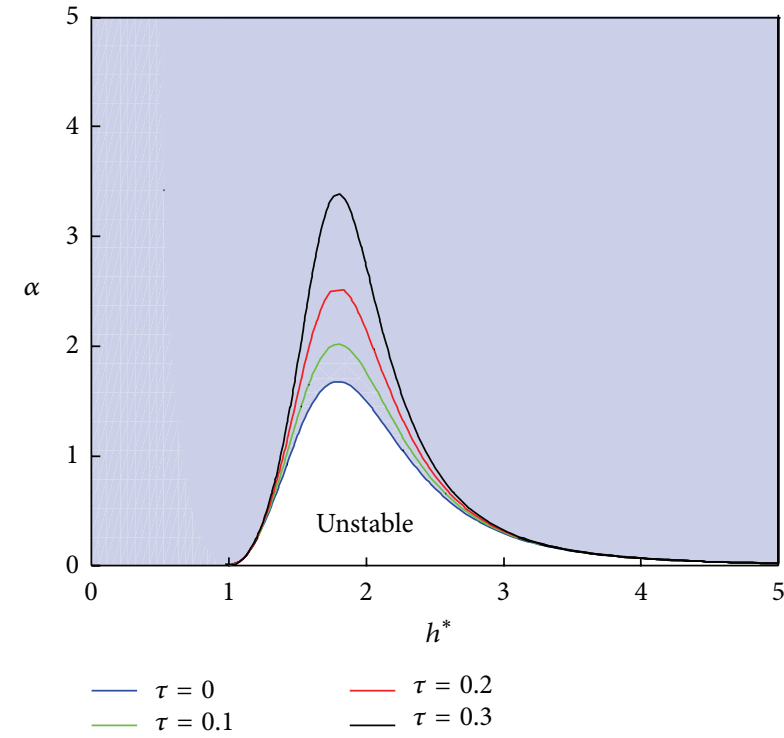

(a)

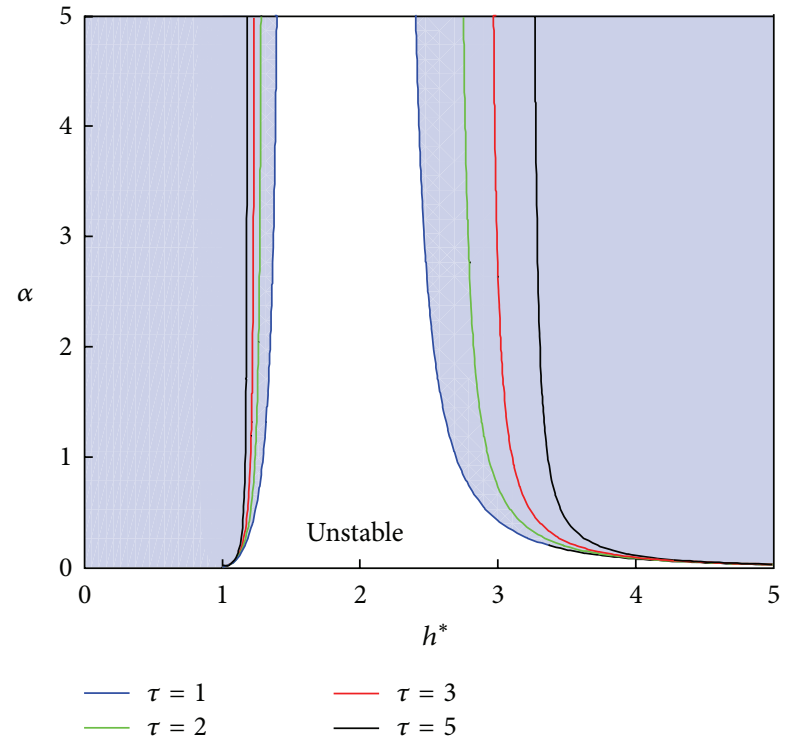

(b)

FIGURE 4: Stability diagram on the $\left(h^{*}, \alpha\right)$ plane on delay impact. (a) $\tau=0,0.1,0.2,0.3$. (b) $\tau=1,2,3,5$.

According to (18), we get the expression about $h_{k}(t-\tau)$

$$
h_{k}(t-\tau)=h_{c}^{*}+\varepsilon \widetilde{h}(Z(t-\tau), T(t-\tau))
$$

By expanding (25) to the fifth order of $\varepsilon$, we get

$$
\begin{aligned}
h_{k}(t-\tau)= & h_{c}^{*}+\varepsilon \tilde{h}-\varepsilon^{2} b \tau \partial_{Z} \tilde{h}+\varepsilon^{3} \frac{(b \tau)^{2}}{2} \partial_{Z}^{2} \widetilde{h} \\
& -\varepsilon^{4} p \tau \partial_{T} \tilde{h}-\varepsilon^{4} \frac{(b \tau)^{3}}{6} \partial_{Z}^{3} \widetilde{h} \\
& +\varepsilon^{5} b p \tau^{2} \partial_{Z, T}^{2} \widetilde{h}+\varepsilon^{5} \frac{(b \tau)^{4}}{24} \partial_{Z}^{4} \widetilde{h} .
\end{aligned}
$$

Let

$$
h_{k}(t-\tau)=h_{c}^{*}+q
$$

where

$$
\begin{aligned}
q= & \varepsilon \widetilde{h}-\varepsilon^{2} b \tau \partial_{Z} \widetilde{h}+\varepsilon^{3} \frac{(b \tau)^{2}}{2} \partial_{Z}^{2} \widetilde{h}-\varepsilon^{4} p \tau \partial_{T} \widetilde{h} \\
& -\varepsilon^{4} \frac{(b \tau)^{3}}{6} \partial_{Z}^{3} \widetilde{h}+\varepsilon^{5} b p \tau^{2} \partial_{Z, T}^{2} \tilde{h}+\varepsilon^{5} \frac{(b \tau)^{4}}{24} \partial_{Z}^{4} \widetilde{h} .
\end{aligned}
$$

We expand the optimal velocity function at the turning point:

$$
\begin{aligned}
& V\left(h_{k}(t-\tau)\right) \\
& =V\left(h_{c}^{*}\right)+V^{\prime}\left(h_{c}^{*}\right) q+\frac{V^{\prime \prime}\left(h_{c}^{*}\right)}{2 !} q^{2} \\
& +\frac{V^{\prime \prime \prime}\left(h_{c}^{*}\right)}{3 !} q^{3}+\frac{V^{(4)}\left(h_{c}^{*}\right)}{4 !} q^{4}+\frac{V^{(5)}\left(h_{c}^{*}\right)}{5 !} q^{5}, \\
& V\left(h_{k}(t-\tau)\right) \\
& =V\left(h_{c}^{*}\right)+\varepsilon \widetilde{h} V^{\prime}\left(h_{c}^{*}\right) \\
& +\varepsilon^{2}\left(\frac{V^{\prime \prime}\left(h_{c}^{*}\right)}{2} \widetilde{h}^{2}-b \tau V^{\prime}\left(h_{c}^{*}\right) \partial_{Z} \tilde{h}\right) \\
& +\varepsilon^{3}\left[\frac{V^{\prime \prime \prime}\left(h_{c}^{*}\right)}{3 !} \widetilde{h}^{3}-V^{\prime \prime}\left(h_{c}^{*}\right) b \tau \widetilde{h} \partial_{Z} \tilde{h}\right. \\
& \left.+\frac{(b \tau)^{2}}{2} V^{\prime}\left(h_{c}^{*}\right) \partial_{Z}^{2} \widetilde{h}\right] \\
& +\varepsilon^{4}\left[-\frac{(b \tau)^{3}}{6} V^{\prime}\left(h_{c}^{*}\right) \partial_{Z}^{3} \widetilde{h}-p \tau V^{\prime}\left(h_{c}^{*}\right) \partial_{T} \widetilde{h}\right. \\
& +\frac{b^{2} \tau^{2}}{2} V^{\prime \prime}\left(h_{c}^{*}\right)\left(\partial_{Z} \widetilde{h}\right)^{2}+\frac{b^{2} \tau^{2}}{2} \widetilde{h} V^{\prime \prime}\left(h_{c}^{*}\right) \partial_{Z}^{2} \widetilde{h} \\
& \left.-\frac{1}{2}(\widetilde{h})^{2} b \tau V^{\prime \prime \prime}\left(h_{c}^{*}\right) \partial_{Z} \widetilde{h}+\frac{V^{(4)}\left(h_{c}^{*}\right)}{4 !} \widetilde{h}^{4}\right]+\cdots .
\end{aligned}
$$




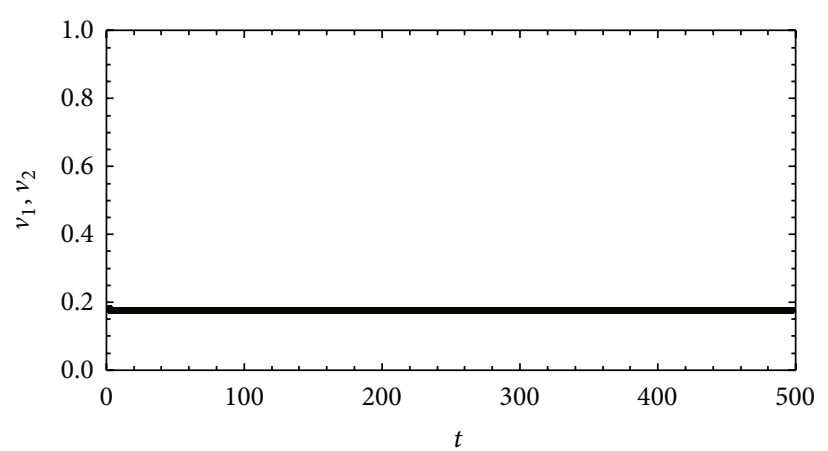

(a)

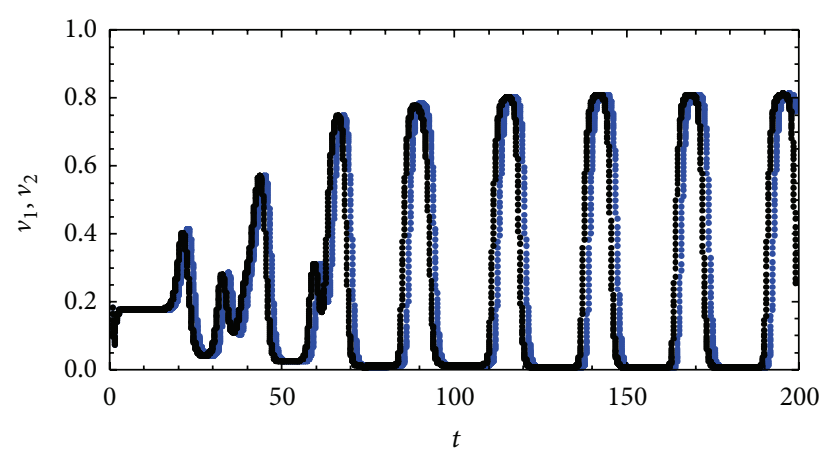

(c)

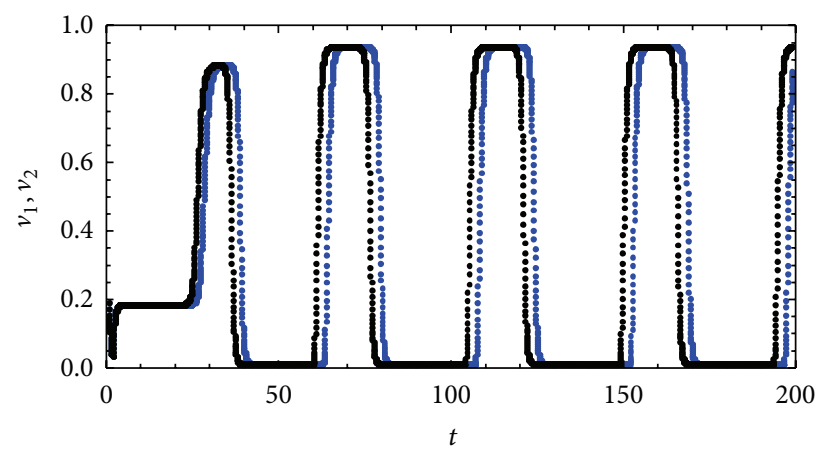

(e)

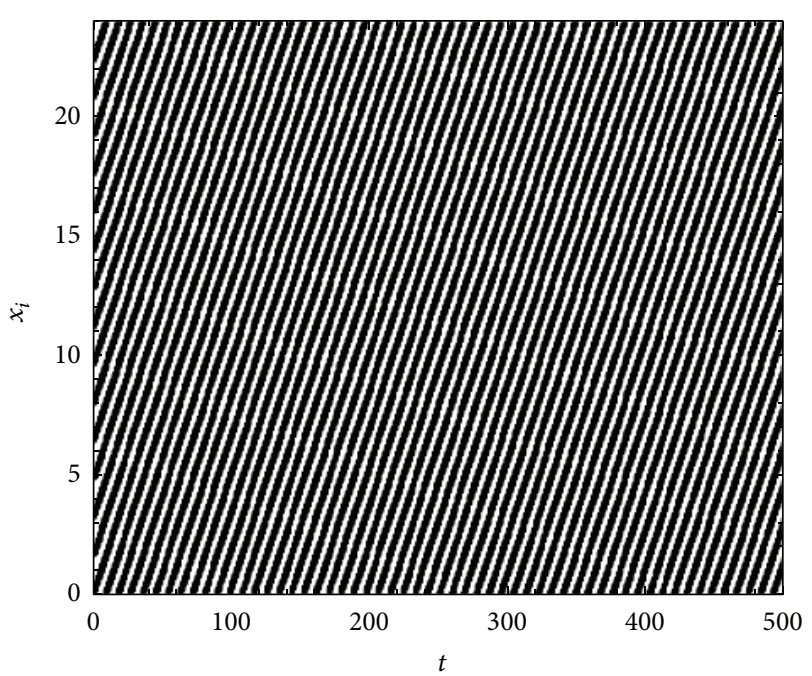

(b)

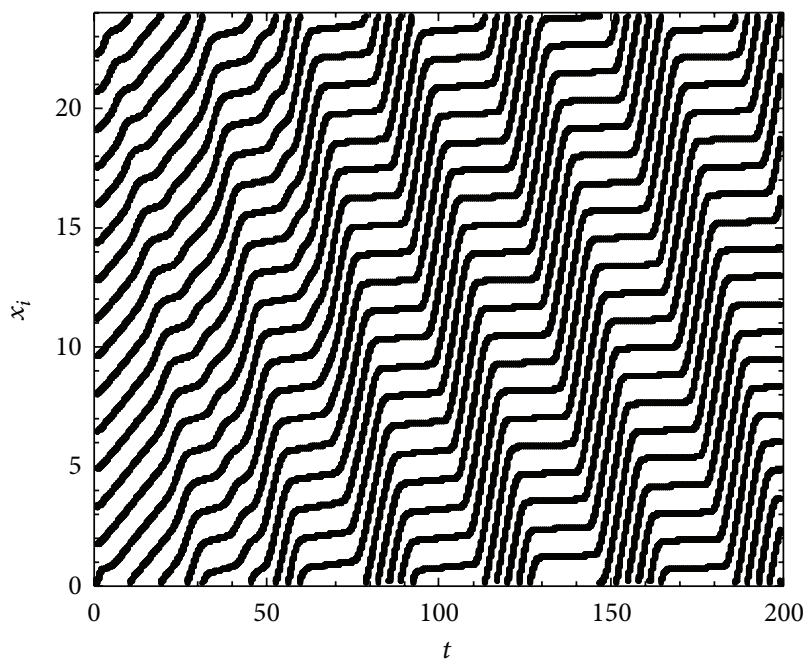

(d)

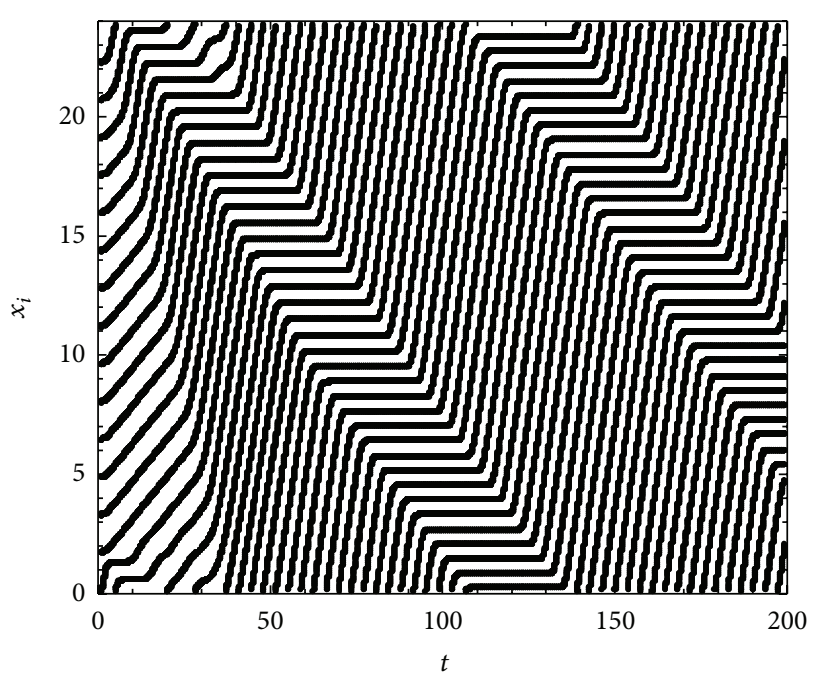

(f)

FIgure 5: Plot of the velocity and the location of vehicles against time in different regions. We consider the speeds of the first two cars and the location of fifteen cars. The blue curve represents the speed of the first car and the black curve represents the speed of the second car. The initial condition is set for $\alpha=2, h^{*}=1.6, v=0.18$. (a) and (b): $\tau=0.2$. (c) and (d): $\tau=0.4$. (e) and (f): $\tau=1$. 
Then

$V\left(h_{k}(t-\tau)\right)-v_{k}=V\left(h_{c}^{*}\right)-\widetilde{v}_{0}+\varepsilon p_{1}+\varepsilon^{2} p_{2}+\varepsilon^{3} p_{3}+\varepsilon^{4} p_{4}$,

where

$$
\begin{gathered}
p_{1}=\widetilde{h} V^{\prime}\left(h_{c}^{*}\right)-\widetilde{h} \widetilde{v}_{1}, \\
p_{2}=\frac{V^{\prime \prime}\left(h_{c}^{*}\right)}{2} \widetilde{h}^{2}-b \tau V^{\prime}\left(h_{c}^{*}\right) \partial_{Z} \widetilde{h}-\widetilde{v}_{20} \widetilde{h}^{2}-\widetilde{v}_{21} \partial_{Z} \widetilde{h}, \\
p_{3}=\frac{V^{\prime \prime \prime}\left(h_{c}^{*}\right)}{3 !} \widetilde{h}^{3}-V^{\prime \prime}\left(h_{c}^{*}\right) b \tau \widetilde{h} \partial_{Z} \widetilde{h}+\frac{(b \tau)^{2}}{2} V^{\prime}\left(h_{c}^{*}\right) \partial_{Z}^{2} \widetilde{h} \\
-\widetilde{v}_{30} \widetilde{h}^{3}-\widetilde{v}_{31} \partial_{Z} \widetilde{h}^{2}-\widetilde{v}_{32} \partial_{Z}^{2} \widetilde{h}, \\
p_{4}=-\frac{(b \tau)^{3}}{6} V^{\prime}\left(h_{c}^{*}\right) \partial_{Z}^{3} \widetilde{h}-p \tau V^{\prime}\left(h_{c}^{*}\right) \partial_{T} \widetilde{h} \\
+\frac{b^{2} \tau^{2}}{2} V^{\prime \prime}\left(h_{c}^{*}\right)\left(\partial_{Z} \widetilde{h}\right)^{2}+\frac{b^{2} \tau^{2}}{2} \widetilde{h} V^{\prime \prime}\left(\bar{h}_{c}\right) \partial_{Z}^{2} \widetilde{h} \\
-\frac{1}{2}(\widetilde{h})^{2} b \tau V^{\prime \prime \prime}\left(h_{c}^{*}\right) \partial_{Z} \widetilde{h}+\frac{V^{(4)}\left(h_{c}^{*}\right)}{4 !} \widetilde{h}^{4}-\widetilde{v}_{40} \widetilde{h}^{4} \\
-\widetilde{v}_{41} \partial_{Z} \widetilde{h}^{3}-\widetilde{v}_{42} \partial_{Z}^{2} \widetilde{h}^{2}-\widetilde{v}_{43} \partial_{Z}^{3} \widetilde{h} .
\end{gathered}
$$

Then, collecting terms of equal order on both sides and comparing coefficients of $\widetilde{h}, \widetilde{h}^{2}, \partial_{Z} \widetilde{h}$, and so forth, we obtain $\widetilde{v}_{n}$ :

$$
\begin{gathered}
\widetilde{v}_{0}=V\left(h_{c}^{*}\right), \\
\widetilde{v}_{1}=V^{\prime}\left(h_{c}^{*}\right), \\
\widetilde{v}_{20}=\frac{V^{\prime \prime}\left(h_{c}^{*}\right)}{2}=0, \\
\widetilde{v}_{21}=-b \tau-\frac{b \widetilde{v}_{1}}{\alpha}, \\
\widetilde{v}_{30}=\frac{V^{\prime \prime \prime}\left(h_{c}^{*}\right)}{6}, \\
\widetilde{v}_{31}=0, \\
\widetilde{v}_{32}=\frac{(b \tau)^{2}}{2} V^{\prime}\left(h_{c}^{*}\right)-\frac{b \widetilde{v}_{21}}{\alpha}, \\
\widetilde{v}_{40}=\frac{V^{(4)}\left(h_{c}^{*}\right)}{4 !}, \\
\widetilde{v}_{41}=-\frac{1}{6} b \tau V^{\prime \prime \prime}\left(h_{c}^{*}\right)-\frac{b \widetilde{v}_{30}}{\alpha}, \\
\widetilde{v}_{42}=0, \\
\widetilde{v}_{43}=-\frac{1}{6}(b \tau)^{3} V^{\prime}\left(h_{c}^{*}\right)-\frac{b \widetilde{v}_{32}}{\alpha} .
\end{gathered}
$$

Substituting $\widetilde{v}$ derived in this way into (7), we get a reduced equation for $\widetilde{h}$ :

$$
\begin{aligned}
\dot{v}_{k}= & \varepsilon^{2} b \partial_{Z} \widetilde{h}+\varepsilon^{4} p \partial_{T} \widetilde{h} \\
= & v_{k+1}-v_{k} \\
= & \widetilde{v}\left(\varepsilon \widetilde{h}(Z+2 \varepsilon, T), \varepsilon^{2} \partial_{Z} \widetilde{h}(Z+2 \varepsilon, T),\right. \\
& \left.\varepsilon^{3} \partial_{Z}^{2} \widetilde{h}(Z+2 \varepsilon, T), \ldots\right) \\
& -\widetilde{v}\left(\varepsilon \widetilde{h}(Z, T), \varepsilon^{2} \partial_{Z} \widetilde{h}(Z, T), \varepsilon^{3} \partial_{Z}^{2} \widetilde{h}(Z, T), \ldots\right) \\
= & \varepsilon^{2}\left[\widetilde{v}_{1} \partial_{Z} \widetilde{h}\right]+\varepsilon^{3}\left[2\left(\widetilde{v}_{1}+\widetilde{v}_{21}\right) \partial_{Z}^{2} \widetilde{h}\right] \\
+ & \varepsilon^{4}\left[\frac{4}{3} \widetilde{v}_{1} \partial_{Z}^{3} \widetilde{h}+2 \widetilde{v}_{21} \partial_{Z}^{3} \widetilde{h}+2 \widetilde{v}_{30} \partial_{Z} \widetilde{h}^{3}\right. \\
& \left.+4 \widetilde{v}_{31}\left(\partial_{Z} \widetilde{h}\right)^{2}+2 \widetilde{v}_{32} \partial_{Z}^{3} \widetilde{h}\right] \\
+ & \varepsilon^{5}\left[\frac{2}{3} \widetilde{v}_{1} \partial_{Z}^{4} \widetilde{h}+\frac{4}{3} \widetilde{v}_{21} \partial_{Z}^{4} \widetilde{h}+\widetilde{v}_{30}\left(6 \widetilde{h}^{2} \partial_{Z}^{2} \widetilde{h}+12 \widetilde{h}\left(\partial_{Z} \widetilde{h}\right)^{2}\right)\right. \\
& +\widetilde{v}_{40} 8 \widetilde{h}^{3} \partial_{Z} \widetilde{h}^{2}+\widetilde{v}_{42}\left(4 \widetilde{h} \partial_{Z}^{2} \widetilde{h}+4\left(\partial_{Z} \widetilde{h}\right)^{2}\right) \\
& \left.+6 \widetilde{v}_{41} \widetilde{h}^{2} \partial_{Z}^{2} \widetilde{h}+2 \widetilde{v}_{43} \partial_{Z}^{4} \widetilde{h}\right] .
\end{aligned}
$$

By taking $b=2 \widetilde{v}_{1}=2 V^{\prime}\left(h_{c}^{*}\right), \tau=1 / 2 V^{\prime}\left(h_{c}^{*}\right)-1 / \alpha$, the second-order term of $\varepsilon$ and the third-order term of $\varepsilon$ are eliminated from (33); then we get

$$
\begin{aligned}
\partial_{T} \widetilde{h}= & \frac{1}{p}\left[\left(\frac{4}{3} \widetilde{v}_{1}+2 \widetilde{v}_{21}+2 \widetilde{v}_{32}\right) \partial_{Z}^{3} \widetilde{h}+2 \widetilde{v}_{30} \partial_{Z} \widetilde{h}^{3}\right] \\
& +\frac{\varepsilon}{p}\left[c_{1} \partial_{Z}^{4} \widetilde{h}+c_{2} \partial_{Z}^{2} \widetilde{h}^{3}+c_{3} \partial_{Z} \widetilde{h}^{4}+c_{4} \partial_{Z} \widetilde{h} \partial_{Z} \widetilde{h}^{2}\right],
\end{aligned}
$$

where

$$
\begin{aligned}
& c_{1}=\frac{(2 / 3) \widetilde{v}_{1}+(4 / 3) \widetilde{v}_{21}+2 \widetilde{v}_{32}+2 \widetilde{v}_{43}}{p}, \\
& c_{2}=\frac{2\left(\widetilde{v}_{30}+\widetilde{v}_{41}\right)}{p}, \quad c_{3}=\frac{2 \widetilde{v}_{40}}{p}, \\
& c_{4}=\frac{6\left(\widetilde{v}_{30}+\widetilde{v}_{41}\right)}{p} .
\end{aligned}
$$

Let

$$
g_{1}=\frac{(4 / 3) \widetilde{v}_{1}+2 \widetilde{v}_{21}+2 \widetilde{v}_{32}}{p}, \quad g_{2}=-\frac{2 \widetilde{v}_{30}}{p}
$$

if we ignore the $O(\varepsilon)$ terms in (34), then (34) is transformed into a $\mathrm{Kdv}$ equation

$$
\partial_{T} \tilde{h}=g_{1} \partial_{Z}^{3} \widetilde{h}-g_{2} \partial_{Z} \widetilde{h}^{3} .
$$


In order to derive the regularized equation, we make the following transformation for (37):

$$
T^{\prime}=g_{1} T, \quad \tilde{h}=\sqrt{\frac{g_{1}}{g_{2}}} \tilde{h}^{\prime} ;
$$

then (37) is transformed into a regularized $\mathrm{Kdv}$ equation

$$
\partial_{T^{\prime}} \tilde{h}^{\prime}=\partial_{Z}^{3} \widetilde{h}^{\prime}-\partial_{Z}\left(\widetilde{h}^{\prime}\right)^{3}
$$

If we ignore the $O(\varepsilon)$ terms in (39), this is just the modified $\mathrm{Kdv}$ equation with a kink solution as the desired solution

$$
\tilde{h}_{0}^{\prime}\left(Z, T^{\prime}\right)=\sqrt{c} \tanh \sqrt{\frac{c}{2}}\left(Z-c T^{\prime}\right)
$$

The selected value of propagation velocity $c$ for the kink solution is determined from the $O(\varepsilon)$ term.

Next, assuming that $\widetilde{h}^{\prime}\left(Z, T^{\prime}\right)=\widetilde{h}_{0}^{\prime}\left(Z, T^{\prime}\right)+\varepsilon \widetilde{h}_{1}^{\prime}\left(Z, T^{\prime}\right)$, we take into account the $O(\varepsilon)$ correction:

$$
\begin{gathered}
O(\varepsilon)=\frac{1}{p}\left[c_{1} \partial_{Z}^{4} \widetilde{h}+c_{2} \partial_{Z}^{2} \widetilde{h}^{3}+c_{3} \partial_{Z} \widetilde{h}^{4}+c_{4} \partial_{Z} \widetilde{h} \partial_{Z} \widetilde{h}^{2}\right], \\
O(\varepsilon)=c_{1}^{\prime} \partial_{Z}^{4} \widetilde{h}^{\prime}+c_{2}^{\prime} \partial_{Z}^{2}\left(\widetilde{h}^{\prime}\right)^{3}+c_{3}^{\prime} \partial_{Z}\left(\widetilde{h}^{\prime}\right)^{4}+c_{4}^{\prime} \partial_{Z} \widetilde{h}^{\prime} \partial_{Z}\left(\widetilde{h}^{\prime}\right)^{2},
\end{gathered}
$$

where

$$
\begin{aligned}
& c_{1}^{\prime}=c_{1} \sqrt{\frac{g_{1}}{g_{2}}}, \quad c_{2}^{\prime}=c_{2}\left(\sqrt{\frac{g_{1}}{g_{2}}}\right)^{3}, \\
& c_{3}^{\prime}=c_{3} \frac{g_{1}^{2}}{g_{2}^{2}}, \quad c_{4}^{\prime}=c_{4}\left(\sqrt{\frac{g_{1}}{g_{2}}}\right)^{3} .
\end{aligned}
$$

In order to determine the selected value of the propagation velocity $c$ for the kink solution (37), it is necessary to satisfy the solvability condition

$$
\begin{aligned}
& \left(\widetilde{h}_{0}^{\prime}\left(Z, T^{\prime}\right), M\left(\widetilde{h}_{0}^{\prime}\left(Z, T^{\prime}\right)\right)\right) \\
& \quad \equiv \int_{-\infty}^{+\infty} d Z \widetilde{h}_{0}^{\prime}\left(Z, T^{\prime}\right) M\left[\widetilde{h}_{0}^{\prime}\left(Z, T^{\prime}\right)\right]=0,
\end{aligned}
$$

where

$$
\begin{aligned}
M\left[\tilde{h}_{0}^{\prime}\left(Z, T^{\prime}\right)\right]= & c_{1}^{\prime} \partial_{Z}^{4} \widetilde{h}^{\prime}+c_{2}^{\prime} \partial_{Z}^{2}\left(\widetilde{h}^{3}\right)^{\prime}+c_{3}^{\prime} \partial_{Z}\left(\widetilde{h}^{4}\right)^{\prime} \\
& +c_{4}^{\prime} \partial_{Z} \tilde{h}^{\prime} \partial_{Z}\left(\widetilde{h}^{2}\right)^{\prime} .
\end{aligned}
$$

Then, we can get

$$
\tilde{h}(Z, T)=\sqrt{\frac{g_{2}}{g_{1}}} c \tanh \sqrt{\frac{c}{2}}\left(Z-c g_{1} T\right) .
$$

\section{Conclusion and Discussion}

In this paper, we study a car-following model with driver's reaction time. First, we investigate the traffic congestion from the view of chaos system synchronization transition. Our result shows that the uniform flow corresponds to the complete synchronization and the stop-and-go congested state corresponds to the lag synchronization of the vehicles. Second, we derive analytical criteria for synchronization manifolds stability; the analytical result and the numerical result are consistent. Third, the synchronization manifold stability regions are further classified; we find that the vehicles exhibit different states of motion in different regions, and the synchronization transition can also be trigged by the driver's reaction time. Last, we analyze the car-following model by the use of the nonlinear analysis method and we derive the modified $\mathrm{KdV}$ equation describing the kink density wave. Our result also shows that it is crucial to pay attention to the behavior of individual drivers in order to understand the emergent behavior of traffic. The driver's reaction time is influenced by individual differences, personality, environment, and other factors. In further work, we need to study how to better characterize the reaction time of the drivers.

\section{Conflict of Interests}

The authors declare that there is no conflict of interests regarding the publication of this paper.

\section{Acknowledgments}

This research was supported by the National Natural Science Foundation of China (Grant no. 11032009) and National Natural Science Foundation of China (Grant no. 11272236).

\section{References}

[1] D. Helbing, "Traffic and related self-driven many-particle systems," Reviews of Modern Physics, vol. 73, no. 4, pp. 1067-1141, 2001.

[2] M. Gerwinski and J. Krug, "Analytic approach to the critical density in cellular automata for traffic flow," Physical Review E, vol. 60, no. 1, pp. 188-196, 1999.

[3] M. Bando, K. Hasebe, A. Nakayama, A. Shibata, and Y. Sugiyama, "Dynamical model of traffic congestion and numerical simulation," Physical Review E, vol. 51, no. 2, pp. 1035-1042, 1995.

[4] M. Bando, K. Hasebe, K. Nakanishi, and A. Nakayama, "Analysis of optimal velocity model with explicit delay," Physical Review E: Statistical Physics, Plasmas, Fluids, and Related Interdisciplinary Topics, vol. 58, no. 5, pp. 5429-5435, 1998.

[5] T. Nagatani, "Bunching of cars in asymmetric exclusion models for freeway traffic," Physical Review E, vol. 51, no. 2, pp. 922-928, 1995.

[6] T. Nagatani and K. Nakanishi, "Delay effect on phase transitions in traffic dynamics," Physical Review E, vol. 57, no. 6, pp. 64156421, 1998.

[7] T. Nagatani, "Thermodynamic theory for the jamming transition in traffic flow," Physical Review E, vol. 58, no. 4, pp. 42714276, 1998. 
[8] T. Nagatani, "Chaotic jam and phase transition in traffic flow with passing," Physical Review E, vol. 60, no. 2, pp. 1535-1541, 1999.

[9] T. Nagatani, "Traffic behavior in a mixture of different vehicles," Physica A: Statistical Mechanics and its Applications, vol. 284, no. 1, pp. 405-420, 2000.

[10] G. Orosz, R. E. Wilson, and B. Krauskopf, "Global bifurcation investigation of an optimal velocity traffic model with driver reaction time," Physical Review E: Statistical, Nonlinear, and Soft Matter Physics, vol. 70, no. 2, Article ID 026207, 10 pages, 2004.

[11] G. Orosz, R. E. Wilson, R. Szalai, and G. Stépán, "Exciting traffic jams: nonlinear phenomena behind traffic jam formation on highways," Physical Review E, vol. 80, no. 4, Article ID 046205, 2009.

[12] G. Orosz, R. E. Wilson, and G. Stépán, "Traffic jams: dynamics and control," Philosophical Transactions of the Royal Society of London A, vol. 368, no. 1928, pp. 4455-4479, 2010.

[13] M. Treiber, A. Hennecke, and D. Helbing, "Congested traffic states in empirical observations and microscopic simulations," Physical Review E, vol. 62, no. 2, pp. 1805-1824, 2000.

[14] D. Helbing, A. Hennecke, and M. Treiber, "Phase diagram and traffic state in the presence of inhomogeneities," Physical Review Letters, vol. 82, pp. 4360-4363, 1999.

[15] B. S. Kerner, The Physics of Traffic, Springer, Berlin, Germany, 2004.

[16] B. S. Kerner and S. L. Klenov, "Deterministic microscopic threephase traffic flow models," Journal of Physics A: Mathematical and General, vol. 39, no. 8, pp. 1775-1809, 2006.

[17] B. S. Kerner and S. L. Klenov, "Microscopic theory of spatialtemporal congested traffic patterns at highway bottlenecks," Physical Review E, vol. 68, no. 3, Article ID 036130, 2003.

[18] B. S. Kerner, S. L. Klenov, A. Hiller, and H. Rehborn, "Microscopic features of moving traffic jams," Physical Review E: Statistical, Nonlinear, and Soft Matter Physics, vol. 73, no. 4, Article ID 046107, 2006.

[19] B. S. Kerner and S. L. Klenov, "Phase transitions in traffic flow on multilane roads," Physical Review E, vol. 80, no. 5, Article ID 056101, 2009.

[20] D. Helbing and M. Treiber, "Gas-kinetic-based traffic model explaining observed hysteretic phase transition," Physical Review Letters, vol. 81, no. 14, pp. 3042-3045, 1998.

[21] M. Treiber and D. Helbing, "Memory effects in microscopic traffic models and wide scattering in flow-density data," Physical Review E: Statistical, Nonlinear, and Soft Matter Physics, vol. 68, no. 4, Article ID 046119, pp. 461191-461198, 2003.

[22] H. K. Lee, R. Barlovic, M. Schreckenberg, and D. Kim, "Mechanical restriction versus human overreaction triggering congested traffic states," Physical Review Letters, vol. 92, Article ID 238702, 2004.

[23] L. C. Davis, "Comment on 'analysis of optimal velocity model with explicit delay," Physical Review E: Statistical, Nonlinear, and Soft Matter Physics, vol. 66, no. 3, Article ID 038101, 2002.

[24] I. Gasser, G. Sirito, and B. Werner, "Bifurcation analysis of a class of 'car following' traffic models," Physica D: Nonlinear Phenomena, vol. 197, no. 3-4, pp. 222-241, 2004.

[25] M. Treiber, A. Kesting, and D. Helbing, "Three-phase traffic theory and two-phase models with a fundamental diagram in the light of empirical stylized facts," Transportation Research B, vol. 44, no. 8-9, pp. 983-1000, 2010.

[26] R. Jiang, Q. Wu, and Z. Zhu, "Full velocity difference model for a car-following theory," Physical Review E, vol. 64, no. 1, Article ID 017101, 2001.
[27] R. Jiang and Q.-S. Wu, "Toward an improvement over KernerKlenov-Wolf three-phase cellular automaton model," Physical Review E, vol. 72, no. 6, Article ID 067103, 2005.

[28] H. Y. Lee, H.-W. Lee, and D. Kim, "Origin of synchronized traffic flow on highways and its dynamic phase transitions," Physical Review Letters, vol. 81, no. 5, pp. 1130-1133, 1998.

[29] S. Boccaletti, J. Kurths, G. Osipov, D. L. Valladares, and C. S. Zhou, "The synchronization of chaotic systems," Physics Reports A, vol. 366, no. 1-2, pp. 1-101, 2002.

[30] A. Balanov, N. Janson, D. Postnov, and O. Sosnovtseva, Synchronization, Springer, Berlin, Germany, 2009.

[31] A. Pikovsky, M. Rosenblum, and J. Kurths, Synchronization-A Universal Concept in Nonlinear Science, Cambridge University Press, Cambridge, UK, 2001.

[32] Y. A. Kuznetsov, Elements of Applied Bifurcation Theory, Springer, Berlin, Germany, 2nd edition, 1997.

[33] J. Hale, Theory of Functional Differential Equations, World Publishing Corporation, Beijing, China, 2003.

[34] P. Hövel, Control of Complex Nonlinear Systems with Delay, Springer, Berlin, Germany, 2010. 


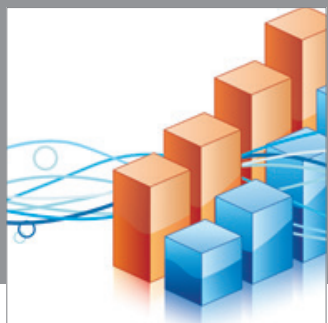

Advances in

Operations Research

mansans

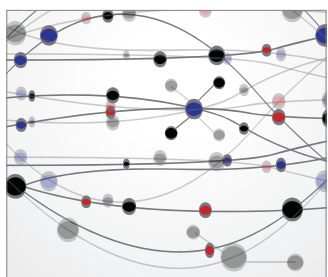

The Scientific World Journal
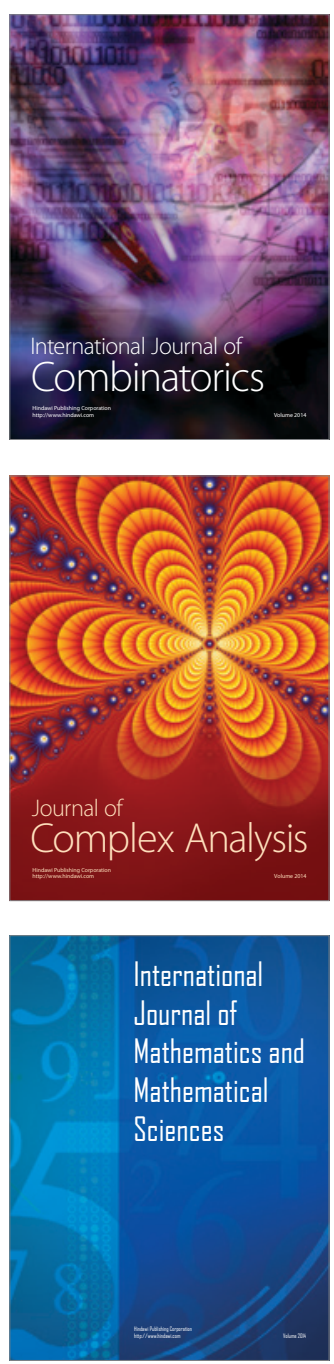
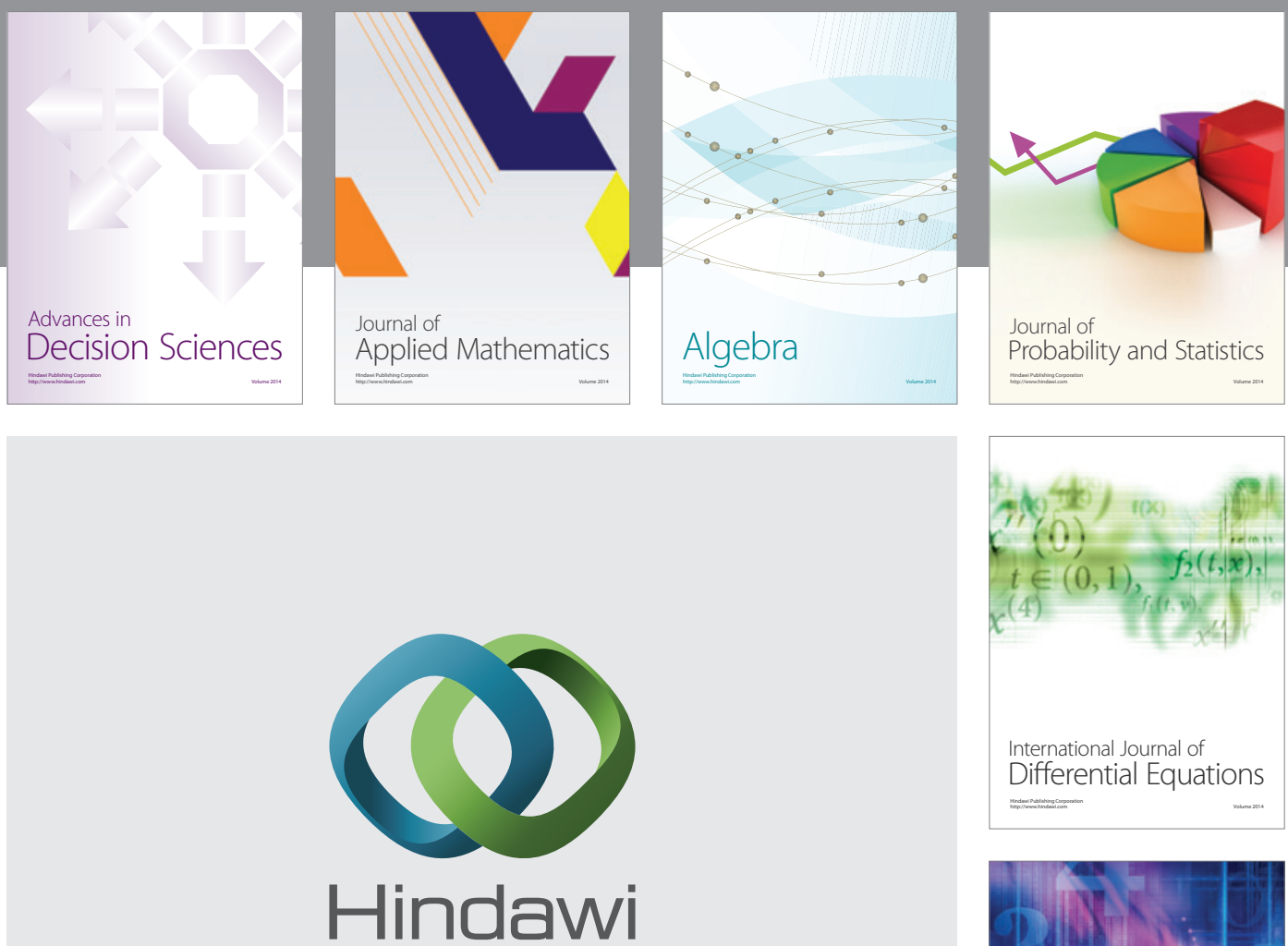

Submit your manuscripts at http://www.hindawi.com
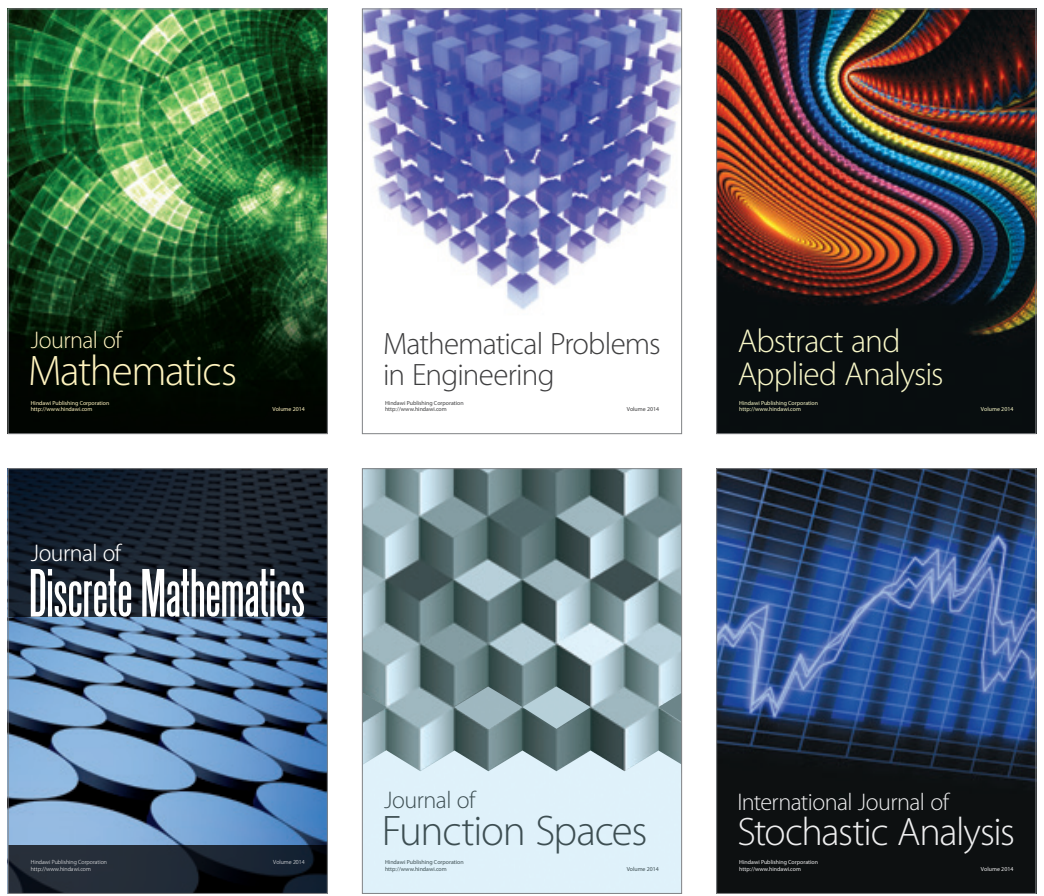

Journal of

Function Spaces

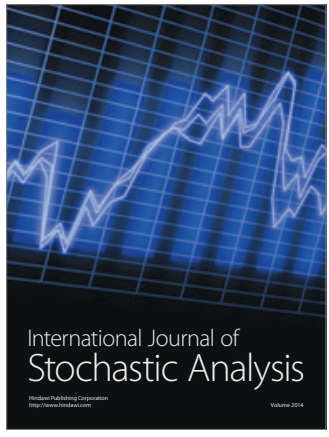

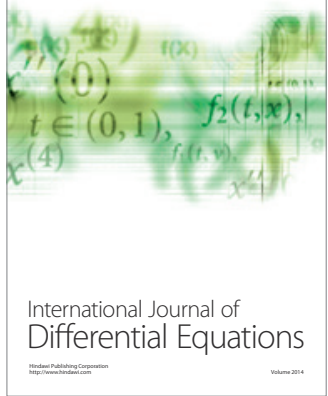
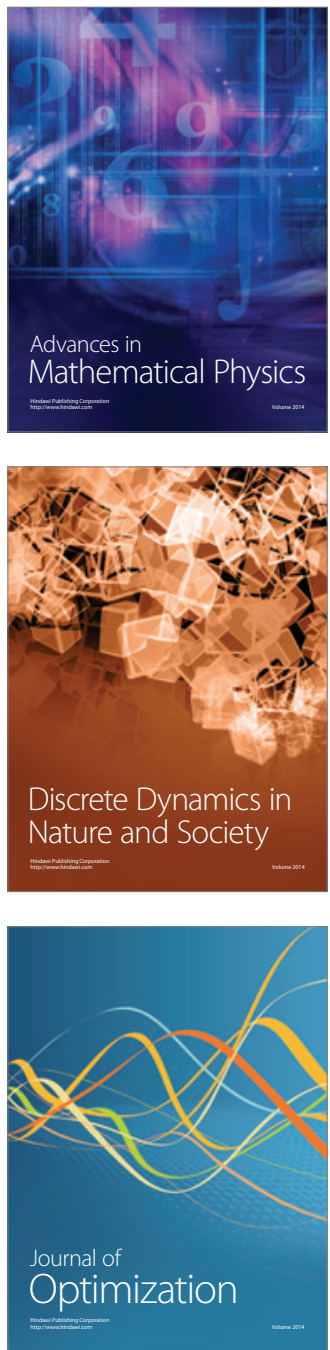